\title{
Squalene synthase promotes the invasion of lung cancer cells via the osteopontin/ERK pathway
}

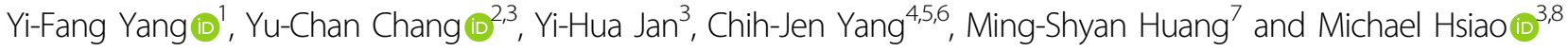

\begin{abstract}
Cholesterol is the major component of lipid rafts. Squalene synthase (SQS) is a cholesterol biosynthase that functions in cholesterol biosynthesis, modulates the formation of lipids rafts and promotes lung cancer metastasis. In this study, we investigated the lipid raft-associated pathway of SQS in lung cancer. Gene expression microarray data revealed the upregulation of secreted phosphoprotein 1 (SPP1; also known as osteopontin, OPN) in CL1-0/SQS-overexpressing cells. Knockdown of OPN in SQS-overexpressing cells inhibits their migration and invasion, whereas an OPN treatment rescues the migration and invasion of SQS knockdown cells. High OPN expression is associated with lymph node status, advanced stage and poor prognosis in patients with lung cancer. Moreover, patients with high SQS expression and high OPN expression show poor survival compared with patients with low SQS expression and low OPN expression. SQS induces the phosphorylation of Src and ERK1/2 via OPN, resulting in increased expression of MMP1 and subsequent metastasis of lung cancer cells. Based on our findings, SQS expression increases the expression of OPN and phosphorylation of Src through cholesterol synthesis to modulate the formation of lipid rafts. SQS may represent a therapeutic strategy for lung cancer.
\end{abstract}

\section{Introduction}

Lung cancer is one of the most common malignancies both in Taiwan and in many other countries. It remains the leading cause of cancer-related death, with more than 1.3 million people dying of the disease annually ${ }^{1}$. Lung cancer is classified according to the histological type into small cell carcinoma and non-small cell lung carcinoma (NSCLC); NSCLC accounts for approximately $80 \%$ of all lung cancer cases $^{2,3}$. Unfortunately, approximately $70 \%$ of all patients who are newly diagnosed with NSCLC present with locally advanced or metastatic disease and require systemic treatment. Patients with NSCLC often have a poor prognosis, and the 5-year survival rate of patients with all stages of the disease combined is only $15 \%^{3-5}$. The high mortality rate of NSCLC is attributed to its high rate

Correspondence: Yu-Chan Chang (jameskobe0@gmail.com) or

Michael Hsiao (mhsiao@gate.sinica.edu.tw)

'Department of Medical Education and Research, Kaohsiung Veterans General Hospital, Kaohsiung, Taiwan

${ }^{2}$ Department of Biomedical Imaging and Radiological Sciences, National YangMing University, Taipei, Taiwan

Full list of author information is available at the end of the article of metastasis ${ }^{6}$. According to previous studies, enzymes involved in the cholesterol biosynthesis pathway are upregulated in lung cancer, and strategies targeting cholesterol biosynthesis enzymes inhibit invasion and migration, indicating that enzymes involved in cholesterol biosynthesis play an important role in cancer progres$\operatorname{sion}^{7}$. Moreover, cholesterol also modulates the formation of lipid rafts (rafts) in the cell membrane. The functions of rafts include the assembly of signaling molecules, modulation of membrane fluidity and membrane protein or receptor exchange ${ }^{8-10}$. Rafts also provide platforms for the coordination of different molecules ${ }^{11,12}$. The cholesterol biosynthesis enzyme squalene synthase (SQS) modulates raft composition and promotes metastasis in lung cancer by inducing clustering of rafts in the cell membrane, leading to an enrichment of the TNF- $\alpha$ receptor in rafts ${ }^{7}$.

Secreted phosphoprotein 1 (SPP1, also known as osteopontin, OPN) was identified and subjected to further analysis due to its ability to promote the invasion and metastasis of various types of cancer ${ }^{13}$. OPN was first 
described as a glycophosphoprotein that is secreted from malignant epithelial cells ${ }^{14}$. The roles of OPN in cancer progression include cell adhesion, chemotaxis, invasion, migration, and anchorage-independent growth of tumor cells $^{15}$. In our previous study, the highest expression of SPP1 was observed in SQS-overexpressing cells ${ }^{7}$. However, the mechanism by which SQS modulates OPN expression to regulate the malignant phenotype has not been clearly elucidated.

\section{Results}

\section{SQS expression is correlated with poor survival rates in lung cancer patients}

In our study, we aimed to identify a key metabolic pathway that is aberrantly overexpressed in invasive lung cancer cell and investigate its role. CL1-1, CL1-2, CL1-3, CL1-4, and CL1-5 were a series of lung cancer cell lines derived from CL1-0 cells and selected by Transwell that had increased invasiveness ${ }^{16}$. In our previous report, we analyzed a gene signature (enzyme annotation) that had differential expression in CL1-0 and CL1-5 (GSE7670) ${ }^{7}$. We identified that the cholesterol biosynthesis pathway was upregulated in highly invasive CL1-5 lung cancer cells. The cholesterol biosynthesis pathway has eight enzymes that were upregulated in CL1-5 cells, including (1) hydroxymethylglutaryl-CoA synthase 1 (HMGCS1), (2) farnesyl-diphosphate synthase (FDPS), (3) farnesyldiphosphate farnesyltransferase (FDFT1, also known as squalene synthase, SQS), (4) squalene epoxidase (SQLE); (5) methylsterol monooxygenase 1 (MSMO1), (6) cytochrome P450, family 51, subfamily A (CYP51A1); (7) 7dehydrocholesterol reductase (DHCR7), and (8) 24dehydrocholesterol reductase $\left(\right.$ DHCR24) (Fig. S1) ${ }^{7}$. Moreover, we evaluated the expression levels of each cholesterol-related enzyme in CL1-0 and CL1-5 cells through a microarray dataset (GSE42407). Based on the results of a statistical analysis of three replicate samples and $P$-values, the expression of $H M G C R, F D F T 1, S Q L E$, MSMO1, DHCR7, and DHCR24 were significantly different between CL1-0 and CL1-5 cells (Fig. 1a). We further

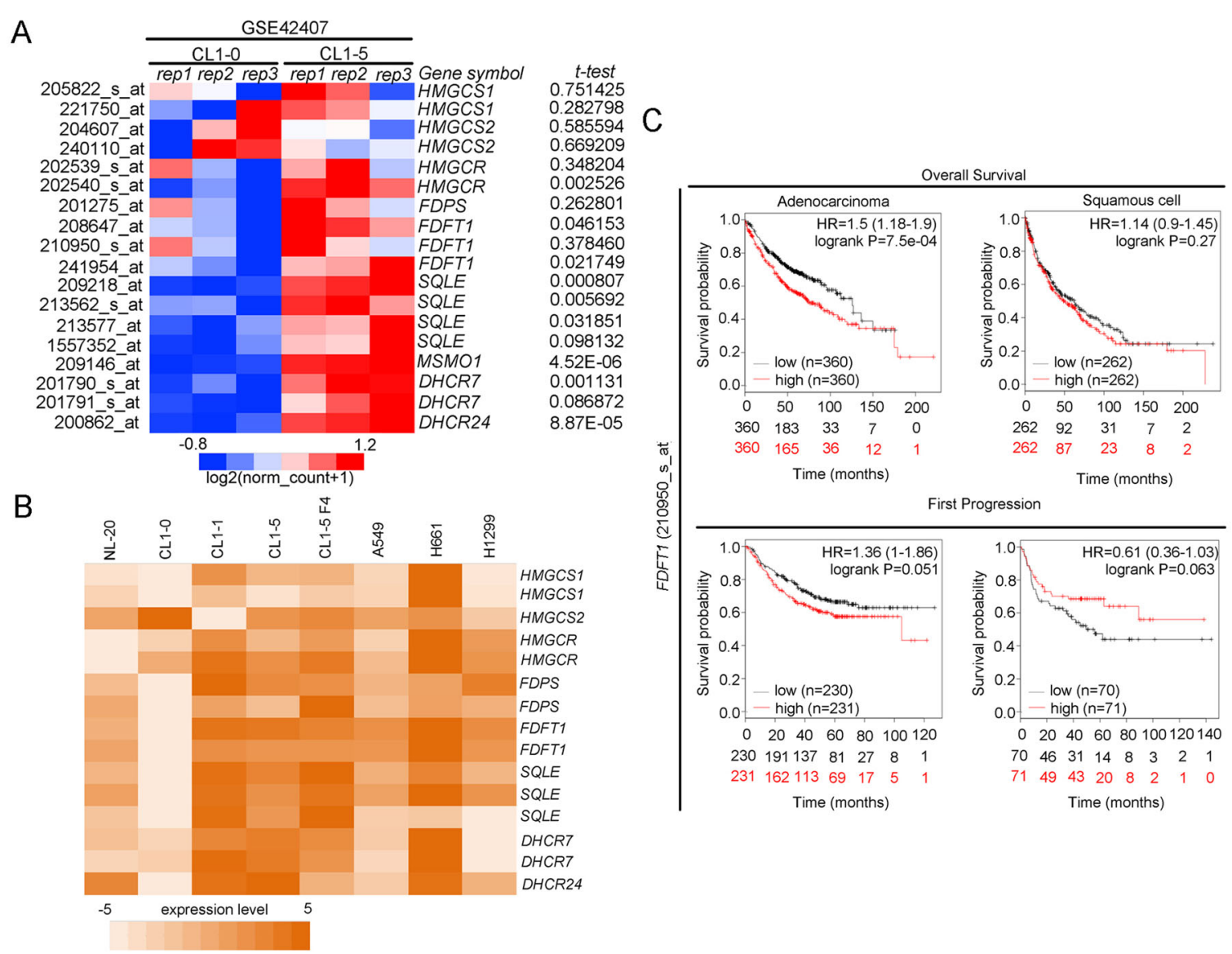

Fig. 1 The cholesterol biosynthesis pathway is upregulated in highly invasive CL1-5 cells. a Expression of each enzyme involved in cholesterol biosynthesis in CL1-0/CL1-5 cells (NCBI/GEO/GSE42407). The significance was determined by Student's $t$ test. $\mathbf{b}$ Expression of each enzyme involved in cholesterol synthesis in various lung cancer cell lines (NCBI/GEO/GSE7670). c The FDFT1 (210950_s_at, SQS) expression level is correlated with the survival curve in patients with lung adenocarcinoma and squamous cell carcinoma subtypes. 
verified the expression levels of each enzyme in a lung cancer cell panel. The heat map shows upregulation of FDFT1 (SQS) in several cell lines compared with the benign and nontumorigenic bronchial epithelial NL-20 cells (Fig. 1b) (GSE7670). Moreover, we detected the expression of the FDFT1 (SQS) gene in a clinical cohort using a specific probe (210950_s_at). A high level of $S Q S$ expression was correlated with the survival rate, including overall survival and survival to the first progression, particularly in patients with the lung adenocarcinoma subtype (Fig. 1c). SQS is the first enzyme of the cholesterol branch that is upregulated in highly invasive lung cancer cell lines. Overexpression of SQS promotes lung cancer invasion and metastasis in vitro and in vivo and enhances cholesterol biosynethsis ${ }^{7}$. Thus, SQS plays an important role in lung cancer progression.

\section{OPN is required for SQS-induced lung cancer cell migration/invasion}

To identify the molecular mechanism and downstream pathways by which SQS regulates lung cancer cell progression, we analyzed the GSE37868 (as an mRNA microarray of CL1-0/SQS compared with CL1-0/Vector) datasets $^{7}$, and secreted phosphoprotein 1 (SPP1; also known as osteopontin, OPN) was the top-ranking downstream factor that was upregulated upon SQS overexpression in CL1-0 cells. Therefore, we evaluated whether SQS regulated OPN expression in SQSoverexpressing cells. A promoter assay, real-time polymerase chain reaction and western blot analysis confirmed that OPN was upregulated in SQS-overexpressing CL1-0 cells compared with the vector control cells (Fig. 2a). Knockdown of SQS significantly decreased expression of OPN in CL1-5 (Fig. 2b). Overexpression of SQS was significantly increased migration and invasion capabilities in CL1-0 cells (Fig. S2A). We further investigate the effect of OPN on migration and invasion abilities of CL1-0/SQS cells. We observed a significant inhibition of migration and invasion following the knockdown of OPN with an shRNA in SQS-overexpressing cells (Fig. 2c and Fig. S2B). Conversely, the replenishment of OPN in SQS knockdown cells significantly restored the invasion and migration of A549 and CL1-5 cells (Fig. 2d, e and Fig. S2C, D).

\section{SQS induces metabolic reprogramming}

Knockdown of SQS reduced the cholesterol levels and inhibited invasion ability in lung cancer cell lines. Furthermore, knockdown of the downstream enzyme of SQS (SQLE, CYP51A1, MSMO1, and DHCR7) also significantly inhibited migration/invasion capabilities compared with the upstream enzyme (HMGCS1 and HMGCR2) in lung cancer cell lines ${ }^{7}$. We determined the metabolic signatures of lung cancer cells from the Cancer Cell Line Encyclopedia (CCLE) database to describe the metabolic events induced by SQS in lung cancer. Robin et al. developed the CERES score to summarize the genelevel dependency from CRISPR-Cas9 knockdown dataset across 342 cancer cell lines ${ }^{17}$. Furthermore, CERES is combined with the metabolic signature to define metabolite-dependent associated with gene levels in cancer cells ${ }^{18}$. Using the metabolite-dependency association algorithm, we noticed substantial changes in the levels multiple metabolites, particularly cholesteryl esters (CE) (Fig. 3a). Previous studies have defined rafts as cholesterol-rich microdomains in the plasma membrane that play a role in a number of signaling processes involving growth factor receptors, $\mathrm{T}$-cell receptors, and the TNF receptor superfamily ${ }^{19-23}$. Silencing the TNF receptor inhibited migration/invasion capabilities in SQSoverexpressing lung cancer cells ${ }^{7}$. Moreover, we dissected the metabolite panels of various lung cancer cells. We analyzed the correlation between SQS expression and the ratio between glycolysis/OXPHOs in NSCLC cell lines $(n=80)$. We stratified cell lines into an SQS-low group $(<70)$ and an SQS-high group $(\geqq 70)$ and observed SQS expression was significantly associated with acetyl-CoA $(P=0.001)$ and citrate $(P=0.01)$ levels (Fig. 3b and Appendix No. 1). Thus, SQS expression is correlated with metabolic signature changes in lung cancer cell lines.

\section{OPN modulates invasion through a cholesterol-dependent increase in ERK phosphorylation}

We postulated that the upregulation of SQS might induce an enrichment and activation of receptors in rafts. Previous studies have shown that Src family kinases (SFKs) are localized in the rafts ${ }^{24}$, and we evaluated the expression of Src pathway-related kinase in CL1-0/SQS cells by western blot analysis. Overexpression of SQS increased the phosphorylation of Src (Tyr416), ERK1/2, and AKT (Ser473), whereas SQS knockdown reduced the phosphorylation of Src, ERK1/2 and AKT in A549 and CL1-5 cells (Fig. 4a, b). Knockdown of OPN significantly decreased the expression of pSrc, pERK1/2, and pAKT in CL1-0/SQS cells (Fig. S3A). We focused on Src in the next experiment because OPN increases the phosphorylation of ERK1/2 and AKT via $\mathrm{Src}^{13}$. Similarly, the KEGG analyzed shows PI3K-AKT and MAPK signaling were upregulated in SQS overexpression cell by using DAVID software (GSE37868, Table S1). We next investigated whether SQS increased the phosphorylation of ERK1/2 and AKT via Src. As shown in Fig. 4c, SQS-induced phosphorylation of Src and ERK1/2 was abolished by an Src inhibitor (Src kinase inhibitor-1, SKI).

We analyzed the levels of proteins involved in these canonical pathways in lung cancer cells using a reversephase protein lysate microarray (RPPA) to confirm that SQS promotes lung cancer metastasis through the $\mathrm{Src} /$ ERK pathway. The phosphorylation of AKT (S473) and 


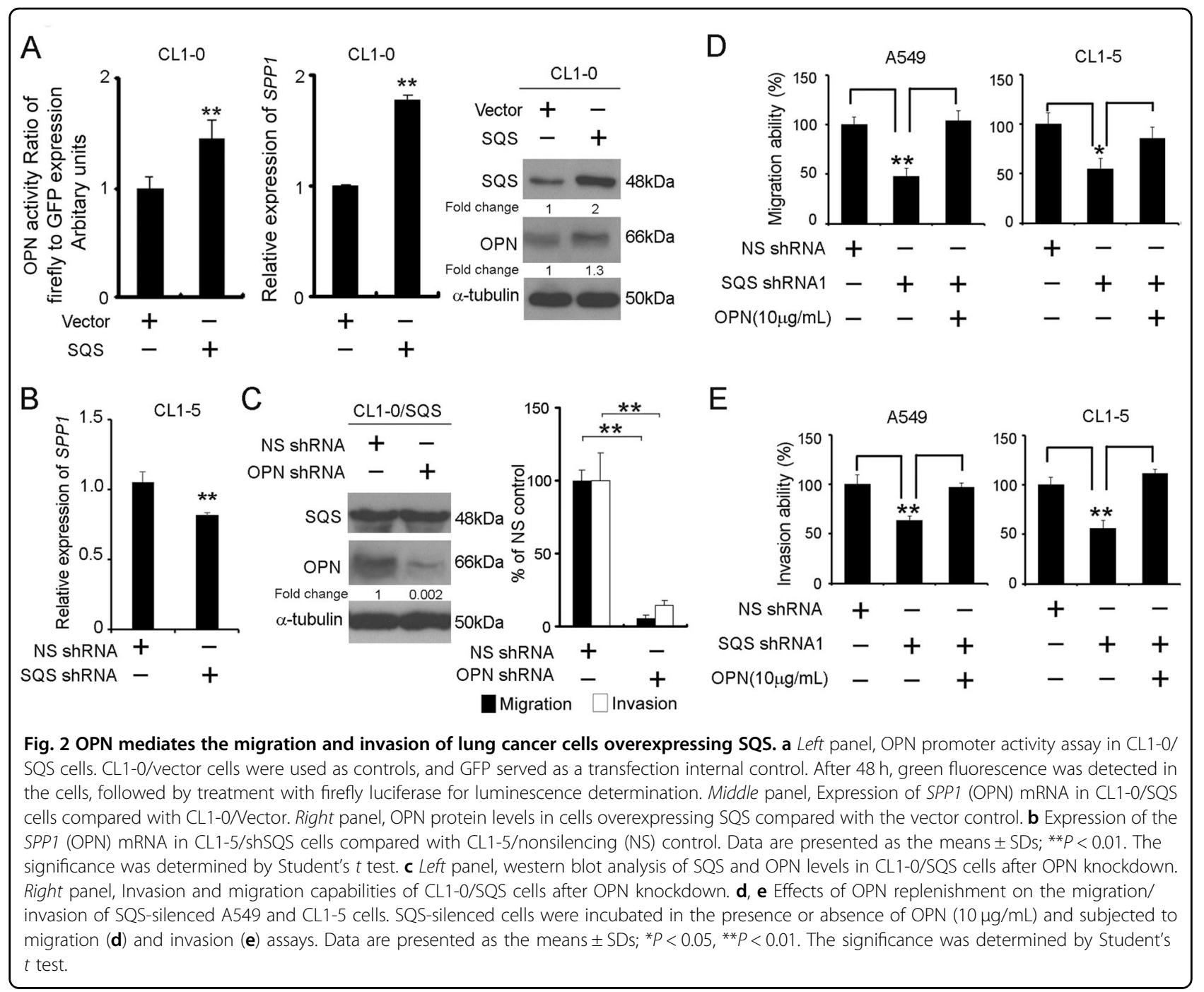

Src (Y416) strongly correlated with the migration ability of lung cancer cell lines (Fig. 4d). We further evaluated the correlations between pAKT and pSrc levels with the migration abilities of lung cancer cell lines. The correlation plots confirmed the positive correlations between pSrc levels with the migration abilities of a lung cancer cell panel (Spearman's $r h o=0.816, P=0.004)$ (Fig. 4e). In addition, we also observed a significant inhibition of the migration and invasion of SQS-overexpressing cells following a treatment with the SKI (Src inhibitor), PD98059 (MEK/ERK inhibitor) and LY294002 (PI3K inhibitor) (Fig. 4f). We examined whether SQS mediated MMP1 expression through Src, ERK, and PI3K in CL1-0/SQS cells. Treatment with SKI, MEK, and PI3K inhibitor reduced the MMP1 expression and inhibited invasion/ migration capabilities in CL1-0/SQS cells (Fig. 4f and Fig. S3B). Next, we investigated whether rafts were involved in the OPN/kinase axis in SQS-overexpressing cells. Treatment with methyl- $\beta$-cyclodextrin $(\mathrm{M} \beta C D$, as a detergent that cholesterol depletes in the rafts), reduced the expression of pSrc, pERK1/2, and pAKT in CL1-0/SQS cells (Fig. 4g). Conversely, cholesterol treatment increased the phosphorylation of Src, ERK1/2, and AKT in CL1-0 cells that typically display a low level of invasion (Fig. 4h). Furthermore, we also observed that CD44 was not involved in the SQS/OPN pathway in SQS-overexpressing cells, in which the cell lines express different levels of CD44 (Fig. S3C, D). Taken together, SQS modulates OPN expression to increase the phosphorylation of Src, ERK1/2 and $\mathrm{AKT}$, and subsequently promotes lung cancer metastasis.

\section{SQS upregulation correlates with OPN expression in lung cancer}

We assessed the expression of SQS/OPN in patients with lung cancer using publicly available cDNA microarray datasets and online survival analysis software to evaluate the ability of the SQS/OPN axis to serve as an 


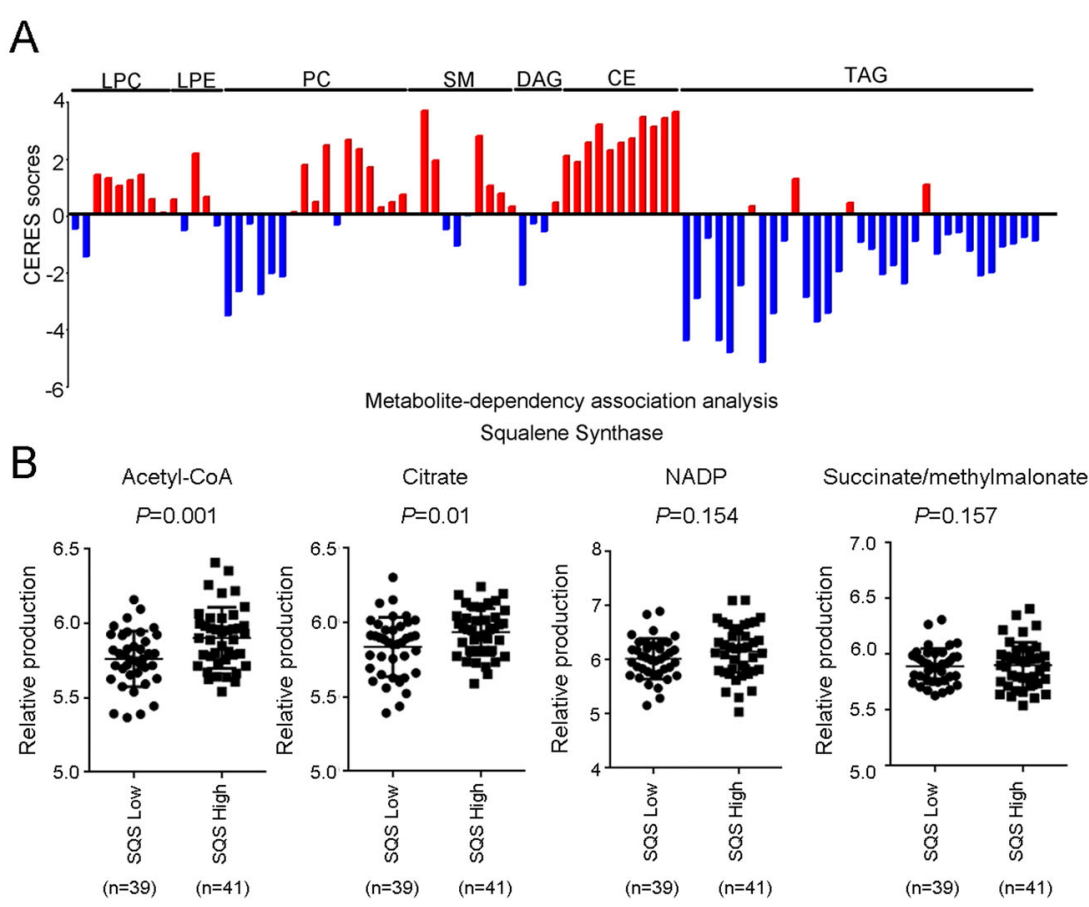

Fig. 3 SQS expression was correlated with changes in metabolic signatures in lung cancer cell lines. a The metabolite-dependency association analysis shows several lipid products in cells with altered SQS expression. LPCs lysophophatidylcholines, LPES lysophosphatidylethanolamines, PCs phosphatidylcholines, SMs sphingomeylins, DAGs diacylglycerols, CEs cholesteryl esters, TAGs triacylglycerols, CERES gene dependencies. $\mathbf{b}$ These swarm plots show several metabolites in lung cancer cell lines with altered SQS expression. The significance was determined by Student's $t$ test.

independent prognostic factor for patients with lung cancer $^{25}$. SPP1 (OPN) was upregulated in lung tumors and associated with a poor overall survival and survival to the first progression (Fig. S4A, B). We further confirmed that the expression of the SQS with OPN mRNAs combined in the clinical cohort exerted a synergistic effect compared with the expression of the OPN mRNA alone. We downloaded the expression levels of $S Q S$ and $O P N$ from the KM plotter website ${ }^{25}$. This metacohort contains several microarray datasets (GSE series), CAARRAY and TCGA. In the clinical part, the website collected the survival time of overall survival (OS) and first progression (FP) of each patient. They further defined the low and high expression levels of $S Q S$ and $O P N$, respectively $(0=$ low, $1=$ high). Therefore, we merged the $S Q S$ and $O P N$ to redefine high (2), others (1) and low (0) to redraw the survival curve by Kaplan-Meier analysis. SQS High/OPN High represented a shorter OS and FP survival compared with SQS Low/OPN Low in patients with lung cancer (Fig. 5a). Moreover, we examined OPN protein expression in lung cancer tissues using immunohistochemical (IHC) staining and correlated OPN expression with the clinicopathological factors of patients with lung cancer. OPN expression was scored according to intensity on a scale ranging from 0-3 points. Using the defined scoring criteria, we stratified scores of 0 and 1 as low OPN expression, and scores of 2 and 3 were categorized as high OPN expression. High OPN expression levels were associated with smoking $(P=0.006)$, the histological type $(P=0.014)$, an advanced stage (stage III-IV, $P=0.005)$ and regional lymph node metastasis $(P=0.024)$ (Table $S 2$ and Fig. S5). Moreover, the survival analysis revealed a significant correlation between high OPN levels and a poor overall survival (OS) and disease-free survival (DFS) compared with low OPN levels (Fig. 5b). The univariate analyses of OS and DFS revealed that OPN expression was a significant predictor of survival (Table S3).

We next investigated whether SQS expression correlated with OPN expression in patients with lung cancer using an IHC analysis and identified a significant positive correlation between SQS and OPN expression (Fig. 5c, Spearman's nonparametric correlation test, correlation coefficient $=0.334, P<0.001$, Table S4). In the analysis of the SQS high/OPN high cohort compared with the SQS low/OPN low cohort, the cumulative survival analysis showed a significant decrease in survival in the high cohort (Fig. 5d). These data suggest that upregulated SQS enhances invasion by upregulated OPN, leading to phosphorylation Src, ERK and AKT, and increased MMP1 expression (Fig. 6). 


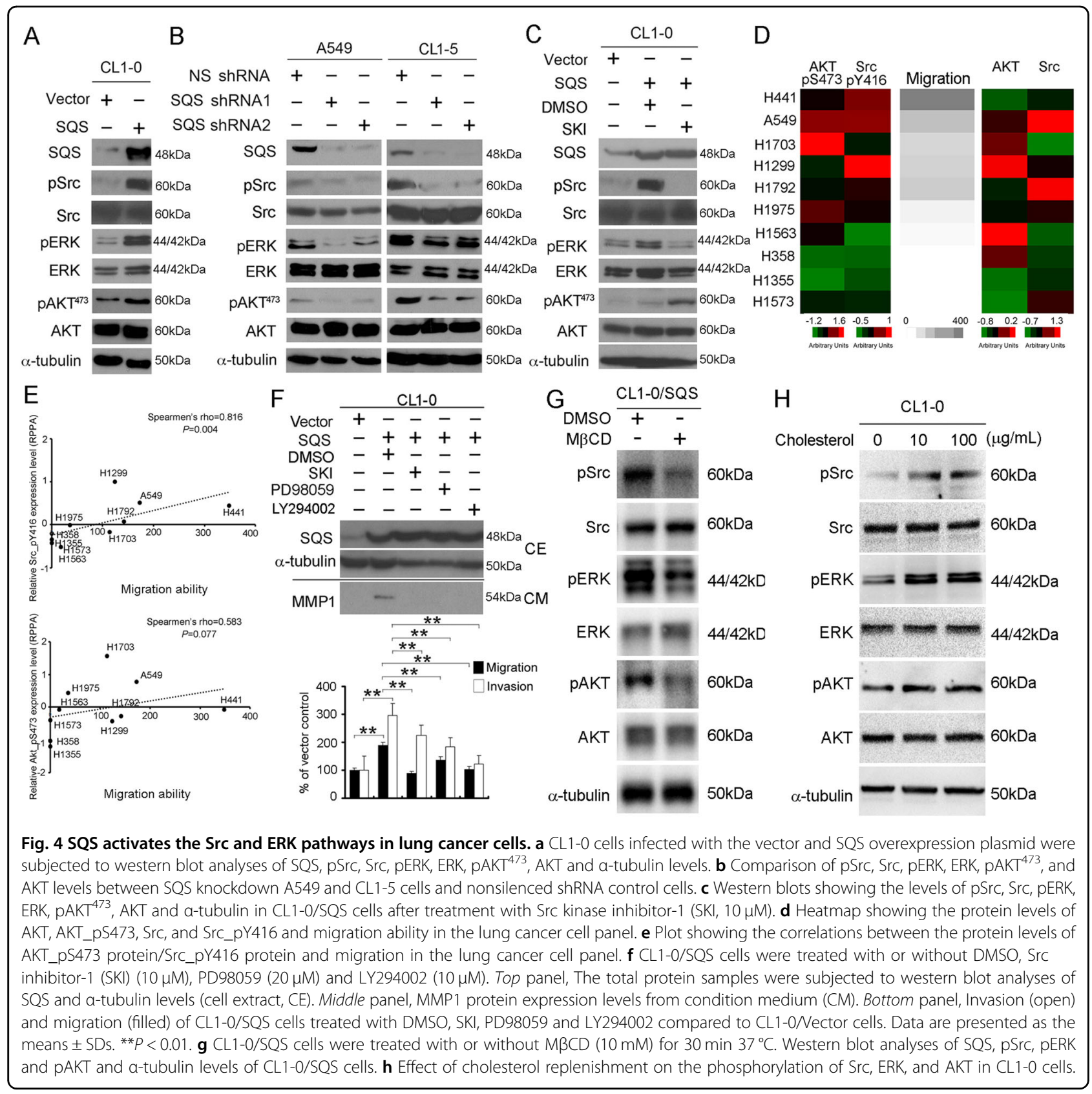

\section{Discussion}

Here, SQS overexpression was associated with a concurrent upregulation of OPN. SQS promotes the invasion and migration of lung cancer cell lines through OPN. According to the results of the clinicopathological analysis, the upregulation of OPN was associated with smoking, histological stage and lymph node metastasis in patients with lung cancer. Moreover, patients with lung cancer presenting with high OPN expression displayed shorter overall survival and disease-free survival (Fig. 5b). SQS induced the phosphorylation of Src, ERK1/2, and AKT in lung cancer cell lines, but the levels of pSrc,
pERK1/2, and MMP1 were decreased after treatment with SKI (Src inhibitor) (Fig. 4c, f). M $\beta C D$ treatment inhibited the phosphorylation of Src, ERK1/2 and AKT in CL1-0/ SQS cells. These findings suggest that SQS promoted invasion/migration abilities via the OPN pathway in lung cancer cells.

OPN is highly expressed in lung antigen-presenting cells (APCs) of mice (smoke-exposed) and is required for the production of IL-17A and smoke-induced emphysema through inhibiting the expression of transcription factor IFN regulator $7(\operatorname{Irf} 7)^{26}$. Moreover, IL-17A regulates tumor growth in lung cancer ${ }^{27}$ and emphysema in patients with 

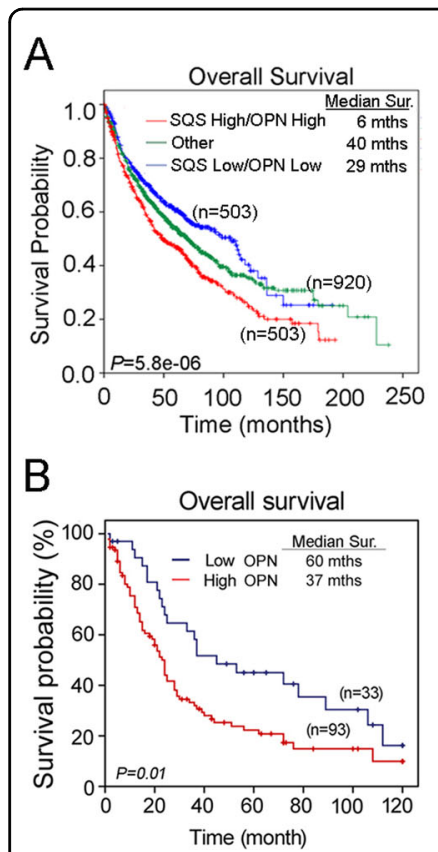
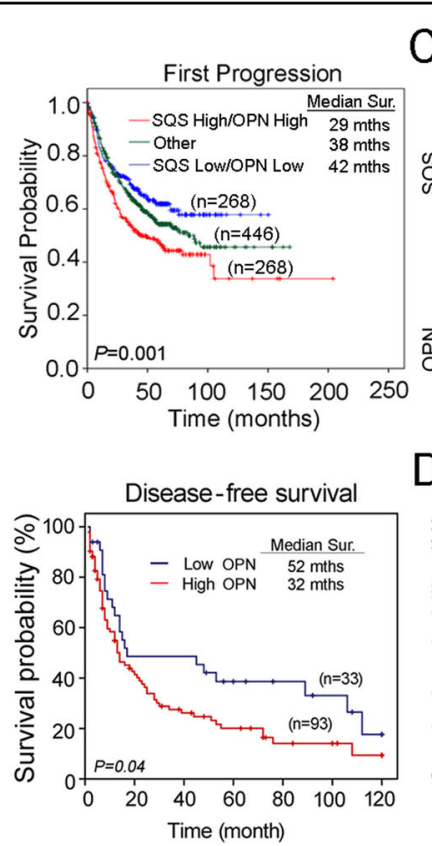

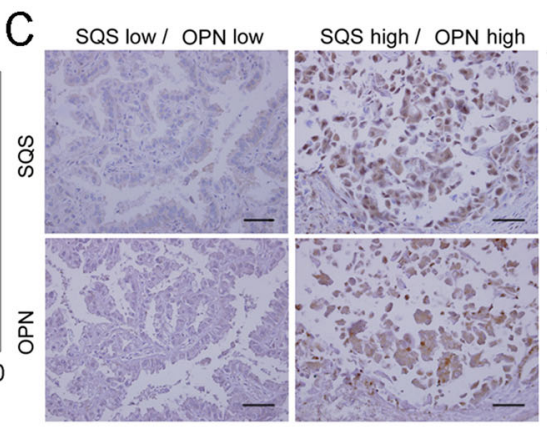

D

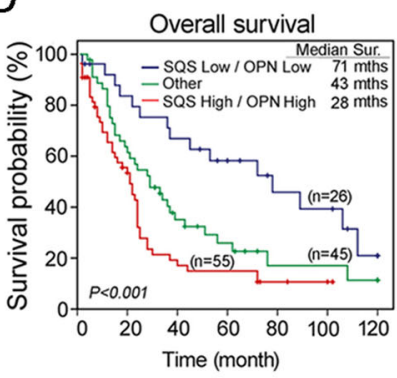

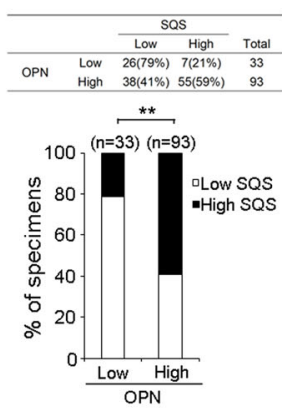

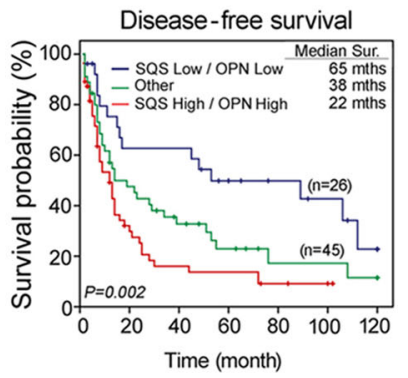

Fig. 5 The expression of genes in the SQS/OPN axis is correlated with poor survival among patients with lung cancer. a Kaplan-Meier analysis of overall survival (left) and survival to the first progression (right) among patients with lung cancer is achieved by examining the combination of SQS and OPN mRNA expression levels. other: SQS Iow/OPN high and SQS high/OPN low. $\mathbf{b}$ Kaplan-Meier analysis of the overall survival (left) and disease-free survival (right) in 126 patients with lung cancer stratified according to SQS and OPN protein levels. c Left, Representative images of SQS and OPN IHC staining in serial sections of lung cancer tissues derived from patients. Right, Quantification of SQS and OPN IHC staining in lung cancer tissues. $N=$ number of specimens. ${ }^{* *} P_{1}<0.01$. Scale bar: $100 \mu \mathrm{m}$. $\mathbf{d}$ Kaplan-Meier analysis of overall survival (left) and disease-free survival (right) in 126 patients with lung cancer stratified according to the SQS and OPN protein levels.

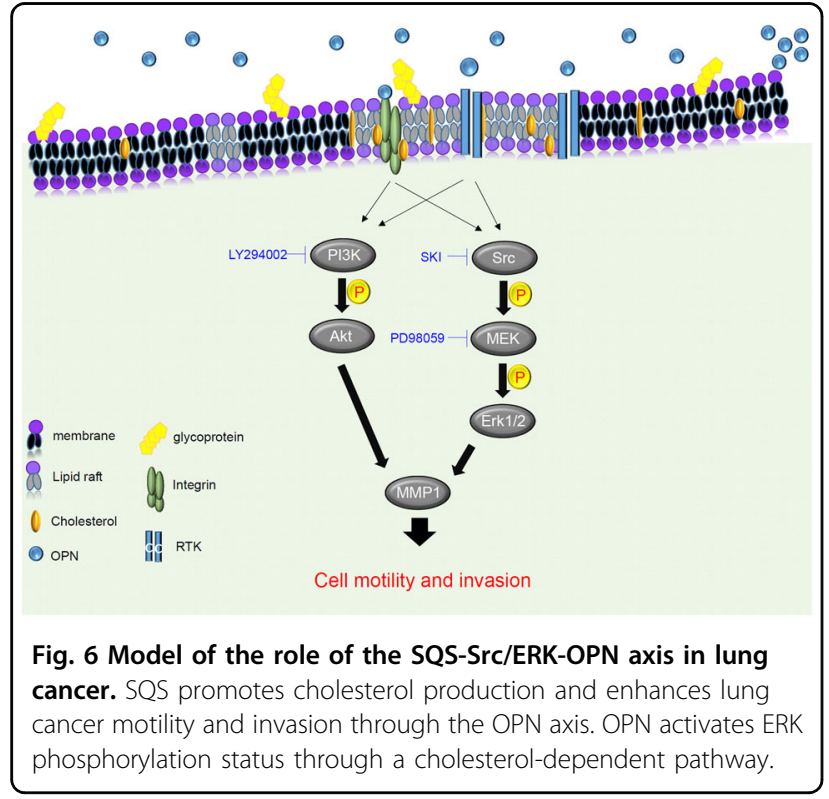

chronic obstructive pulmonary disease (COPD), which is related to lung cancer development ${ }^{28}$. Overexpression of OPN has been reported to be associated with poor survival of patients with lung cancer $^{29}$. The prognostic significance of circulating OPN levels has been reported in patients with lung cancer, in whom elevated plasma OPN levels are associated with disease recurrence after tumor resection ${ }^{30}$. In addition, TNF- $\alpha$-induced OPN expression in $\mathrm{HN}-22$ cells growing on fibronectin supports occurs via $\beta 1$ integrin and $\mathrm{ERK}^{31}$; in the present study, OPN was upregulated in SQS-overexpressing cells and correlated with smoking status in lung cancer patients (Fig. 2a and Table S2). In our previous reports, we found that SQS facilitates TNFR1 enrichment into rafts to enhance lung cancer migration/invasion through the NF$\kappa \mathrm{B}-\mathrm{MMP} 1$ axis $^{11}$. In this study, we discovered other features of SQS. The production of ceramide (CE) and phosphatidylcholine (PC) were upregulated in high SQSexpressing cell lines (Fig. 3a). SQS gene is not only involved in raft remodeling but also regulates the cellular microenvironment.

OPN binds to integrins and CD44 and contains a protease-hypersensitive site $^{32}$. OPN interacts with integrins to induce the activation of phosphoinositide 3-kinase (PI-3Kinase), mitogen-activated protein kinase (MAPK), phospholipase $\mathrm{C}-\gamma$ and protein kinase $\mathrm{C}$ through the c-Src-dependent transactivation of the epidermal growth factor receptor (EGFR) in cancer cells ${ }^{33,34}$. Furthermore, targeting ERK with U0126 (ERK1/2-specific MEK1/2 
inhibitor) reduced OPN expression in MC3T3-E1 cells but not p38 and JNK kinase ${ }^{35}$. Mouse OPN promoter was stimulated by v-Src in HT1080 cells ${ }^{36}$. However, OPN expression did not change with LY294002 (PI3K inhibitor) treatment in MHCC-97L cells ${ }^{37}$. In the present study, SQS overexpression increased OPN, pSrc, pERK1/2 and $\mathrm{pAKT}^{473}$ levels in lung cancer cells, whereas knockdown of SQS or treatment with SKI significantly reduced the levels of pSrc and pERK1/2 (Fig. 4a-c). Similar to our results, Chitose et al. showed that Src activation was limited by cholesterol-enriched raft microdomains ${ }^{38}$. In recent years, Holzer et al. mentioned that Src is necessary for JNK activation to catalyze saturated fatty acids ${ }^{39}$. Saturated fatty acid conditions include type II diabetes and insulin resistance. In several cancer types, fatty acids are also increased through metabolic reprogramming. In addition, it has been claimed that the aggregation of rafts is activated by $\mathrm{Src}$ in several cancer types ${ }^{40,41}$. In this manuscript, we provide many clues consistent with these references. These views reflect the importance of rafts and are related to typical oncogenic pathways.

Previous studies have shown suppression of Src family kinase blocks epidermal growth factor-induced phosphorylation of AKT in T47D breast cancer cells. However, Src inhibitor (PP1) inhibits EGF-induced phosphorylation of AKT in T47D cells at $15 \mathrm{~min}$, and pAKT recovered after treatment for $30 \mathrm{~min}^{42}$. In our studies, we treated Src inhibitors in CL1-0/SQS cell for $16 \mathrm{~h}$ (Fig. 4c). The ending point was referring to the MMP1 study by Yukihiro Hojo et al. ${ }^{43}$. Therefore, we speculate that pAKT has resumed its expression after a long period of drug treatment. Expression of pAKT was decreased after treatment with $\mathrm{M} \beta C D$ in CL1-0/SQS cells (Fig. 4g). Furthermore, SQS mRNA levels were significantly upregulated in lung cancer patients with KRAS mutations ${ }^{7}$. Ras has also been observed to activate and interact with the catalytic subunit of type I phosphatidylinositol-3-kinase (PI3Ks), leading to PI3K translocation to the cell membrane and conformational changes by activation of lipid kinase ${ }^{44,45}$. This is the first study showing that overexpression of SQS increased the expression of pSrc, pERK and pAKT in CL1-0 cells. However, the detailed mechanism by which SQS promotes the expression of pAKT is worthy of further investigation.

Knockdown of SQS destroyed rafts in lung cancer cell lines ${ }^{7}$. CD44 shedding and the delocalization of focal adhesion complexes from rafts induced by cholesterol depletion inhibited tumor cell migration ${ }^{46,47}$. The OPNCD44 interaction contributes to the survival of BA/F3 cells by activating PI3K-AKT signaling ${ }^{48}$. Exogenous OPN treatment increases $\mathrm{CD} 44$ expression and osteoclast motility in $\mathrm{OPN}^{-1-}$ osteoclasts ${ }^{49}$. OPN is upregulated in patients with lung cancer ${ }^{50}$ and interacts with splice variants of CD44 (CD44v) to promote the enrichment of CD44 in rafts, leading to increased survival rates in human cancer cell lines ${ }^{51,52}$. Here, we showed that overexpression of SQS increased pERK and PAKT in CL1-0 and H1355 cells, which have different levels of CD44 (Fig. 4a, Fig. S3C, D). These data suggested that CD44 was not involved in the SQS/OPN pathway. Cholesterol depletion leads to reduced phosphorylation of Src, ERK1/2, and AKT in CL1-0/SQS cells (Fig. 4g). Conversely, cholesterol replenishment induced the phosphorylation of Src, ERK1/ 2 and AKT in CL1-0 cells (Fig. 4h). Although we were unable to exclude the involvement of other pathways in Src phosphorylation, this study is the first to show that SQS overexpression increased pERK levels through Src.

Accumulating evidence has revealed a function for OPN in modulating tumor metastasis ${ }^{53,54}$. Recent reports have indicated that OPN activity is regulated by HIF-1 $\alpha$, is PI3K/AKT pathway-dependent and also accompanies $\mathrm{EMT}^{55,56}$. Moreover, OPN can directly interact with various membrane receptors, including EGFR, CD44, VEGF and integrins, thereby promoting cancer metastasis ${ }^{57,58}$. In our study, OPN knockdown significantly inhibited the migration and invasion of CL1-0/SQS cells, whereas OPN replenishment restored the migration and invasion of A549/shSQS and CL1-5/shSQS cells (Fig. 2c-e). According to a previous study, OPN induced MMP1 expression in smooth muscle cells through integrin $\alpha \mathrm{v} \beta 3^{59}$. In our study, the migration and invasion of SQS-overexpressing cells were inhibited after treatment with Src (SKI), MEK (PD98059), and PI3K (LY294002) inhibitors. The SKI, MEK, and PI3K inhibitors also reduced MMP1 expression in SQS-overexpressing cells (Fig. 4f and Fig. S3B). Based on these findings, we surmised that SQS is required for OPN expression and subsequently promotes the migration and invasion of lung cancer. In this manuscript, we describe this new mechanism and the clinical outcomes of alterations in the SQS/OPN axis in lung cancer. Moreover, we screened these combined approaches in various cancer cell lines. As shown in the survival plots, breast, ovarian and gastric cancers exhibited similar trends (Fig. S6). In our future prospective study, we will develop and implement these strategies for modulating the SQS/OPN axis and expand them to multiple cancer types. We will then perform a clinical study to analyze the correlations with clinicopathological factors. In addition, we will consider combining options as a powerful therapeutic strategy or a prognostic/diagnostic approach for patients with cancer.

In conclusion, this study is the first to investigate the mechanism by which SQS promotes the migration and invasion capabilities of CL1-0 lung cancer cells through the OPN-Src-ERK pathway and its implications for the clinical setting. SQS expression was correlated with reduced overall survival and first progression survival, indicating that SQS is an independent prognostic factor. The combined expression of SQS and OPN could provide a prognostic biomarker for lung cancer metastasis. 


\section{Materials and methods}

\section{Cell lines and cell culture conditions}

The cell lines CL1-0/Vector, CL1-0/SQS, CL1-5-nonsilence, CL1-5-shSQS-1 and CL1-5-shSQS-2 were maintained in RPMI supplemented with 10\% FBS and 1\% penicillin-streptomycin-glutamine (PSG). A549-nonsilence, A549-shSQS-1 and A549-shSQS-2 cells were maintained in DMEM containing 10\% FBS and 1\% PSG. SQS overexpression and knockdown were performed as described previously ${ }^{7}$. The SQS overexpression system was a pLenti-6.3/V5-DEST ${ }^{\mathrm{TM}}$ vector (\#V53306, ThermoFish), and the knockdown system was a pGIPZ shRNA vector (Open Biosystems) or pLKO.1 TRC cloning vector (National RNAi Core Facility Platform). The shRNA sequences are shown in Supplementary Table S5. Src Inhibitor-1 (SKI) (Cat. S2075, $10 \mu \mathrm{M}$ ), PD-98059 (a mitogen-activated protein (MAP) kinase inhibitor, Cat. P215, $20 \mu \mathrm{M}$ ) and cholesterol (Cat.C3045) were purchased from Sigma, St. Louis, MO, USA. All inhibitors were dissolved in DMSO. Cholesterol was dissolved in ethanol.

\section{Western blot analyses}

Western blot analyses were performed using a previously method ${ }^{7}$. The antibodies were directed against SQS (1:2000, GTX104091, Genetex), OPN (1:1000, \#18625, IBL), pSrc (1:1000, \#2101, Cell Signaling Technology), Src (1:1000, \#2109, Cell Signaling Technology), pERK (1:1000, \#9101, Cell Signaling Technology), ERK (1:1000, \#9102, Cell Signaling Technology), pAKT (1:1000, \#4060, Cell Signaling Technology), AKT (1:1000, \#4691, Cell Signaling Technology), MMP1 (1:1000, \#10371-2-AP, Proteintech) and $\alpha$-tubulin (1:10,000, \#T5168, Sigma-Aldrich) (Supplementary Table S5). All western blot data are representative of at least three independent replicates.

\section{Promoter assay}

For the OPN promoter assay, the pGL3-Basic-OPN (containing a fragment of the OPN promoter from bases -2267 to -1 ) plasmid used to detect the transcription of OPN was kindly provided by Dr. Pei-Jung $\mathrm{Lu}^{60}$. The pGL3-Basic plasmid served as a control, and pZsGreen $\mathrm{N} 1$ was used as an internal control for OPN transcription and transfection efficiency. Cells (5000 cells/well) were seeded in 96-well plates and incubated at $37{ }^{\circ} \mathrm{C}$ with $5 \% \mathrm{CO}_{2}$, overnight. The CL1-0/Vector and CL1-0/ SQS cells were transfected with $150 \mathrm{ng}$ pGL3-Basic-OPN and $15 \mathrm{ng}$ pZsGreen $\mathrm{N} 1$ by using X-tremeGENE HP DNA transfection reagent (Roche). After $48 \mathrm{~h}$, the green fluorescence (as transfection control) and luciferase signals were measured by using a plate reader (Victor3, PerkinElmer) and ONE-Glo Luciferase assay (Promega, \#E6120).

\section{Quantitative real-time PCR}

Total RNA was extracted using $\mathrm{TRIzol}^{\mathrm{M}}$ reagent (\#15596026, Thermo Fisher), and cDNAs were synthesized using the SuperScript ${ }^{\mathrm{TM}}$ IV First-Strand Synthesis System (\#18091200, Thermo Fisher). Quantitative realtime PCR was performed using the SYBR Green PCR Supermix (Bio-Rad). The primer sequences are shown in Supplementary Table S5.

\section{Cell migration/invasion assay and OPN replenishment}

The invasion and migration assays were performed as previously described ${ }^{7}$. For OPN replenishment, cells were resuspended in serum-free medium containing OPN $(10 \mu \mathrm{g} / \mathrm{mL}$, \#120-35, PEPROTECH, Rocky Hill, NJ) and added to the upper compartment of each well. All experiments were performed in quadruplicate.

\section{Conditioned medium collection}

The cells were plated on 6 -cm culture plates at a density of $1 \times 10^{6}$ cells in complete medium, incubated for $24 \mathrm{~h}$, and then washed twice with serum-free medium. For the collection of conditioned medium, cells were incubated with or without different inhibitors in $2 \mathrm{~mL}$ of serum-free medium for $24 \mathrm{~h}$. Next, the medium was collected and concentrated $^{61}$.

\section{Cholesterol depletion}

CL1-0/SQS cells were plated at a density of $2 \times 10^{6}$ cells in 10 -dish plates in $10 \%$ FBS complete medium for $24 \mathrm{~h}$ at $37^{\circ} \mathrm{C}$. After $24 \mathrm{~h}$, the CL1-0/SQS cells were washed twice with $1 \%$ FBS medium and then treated with or without $\mathrm{M} \beta \mathrm{CD}(10 \mathrm{mM})$ for $30 \mathrm{~min}$ at $37^{\circ} \mathrm{C}$.

\section{In silico study}

The clinical information and genomics matrix file of The Cancer Genome Atlas (TCGA) database (https:// xenabrowser.net/heatmap/) were download from the USCS Xena browser website. All Gene Expression Omnibus Series (GSE) series datasets downloaded from Gene Express Omnibus (GEO) website were normalized and analyzed using GeneSpring software (Version 13.1.1., Agilent, Santa Clara, CA, USA) and the Kaplan-Meier plotter website (https://kmplot.com/analysis/). All of the data we downloaded from the browser included clinical parameters and expression levels of target genes in patients with lung cancer. This website applied microarray analysis or next-generation sequencing of each probe after normalization. High expression is defined as expression above the median level. In addition, we removed several clinical cases that lacked the corresponding parameters. We further divided the samples into two subtypes of lung cancer: lung adenocarcinoma and lung squamous cell carcinoma. 


\section{In silico datasets and biostatistical analysis}

For Fig. 1a, we downloaded GSE42407 from the GEO website. After normalization, we output all probes and selected cholesterol-related genes for heatmap analysis. Moreover, we calculated the p-value of each probe in the CL1-0 and CL1-5 groups $(n=3)$ through Student's $t$ test. Similarly, we downloaded GSE7670 from the GEO website, retained the lung cancer cell panel, performed the same procedures as in Fig. 1a, and plotted the heatmap in Fig. 1b. From RPPA (reverse-phase protein arrays) profiles from CCLE (Cancer Cell Line Encyclopedia, CCLE_RPPA_20181003.csv, https://portals.broadinstitute. org/ccle/data), we picked target events including Akt, Akt_pS473, Src, and Src_pY416 to compare with our available migration ability through Boyden's chamber. Combining all the evidence, we evaluated these data with heatmaps (Fig. 4d) and correlation plots (Fig. 4e).

\section{Metabolism analysis}

In Fig. 3b, we analyzed the correlation between SQS expression and the ratio between glycolysis/OXPHOs in NSCLC cell lines $(n=80)$. We recorded the expression levels of FDFT1 (SQS) and several glycolysis/OXPHOsrelated products, including $\alpha$-ketoglutarate, AMP, citrate, isocitrate, DHAP/glyceraldehyde 3P, F1P/F6P/G1P/G6P, fumarate/maleate/alpha- ketoisovalerate, lactate, malate, NAP, NADP, oxalate, PEP and succinate/methylmalonate (Appendix No. 1).

\section{KEGG analysis}

DAVID Functional Annotation Bioinformatics Microarray Analysis is a prediction software that can be used for transcriptome datasets ${ }^{62}$. We set the threshold to $>1.5$ fold change and exported these probe lists from GeneSpring software. We further converted the gene ID list into gene symbols on the DAVID website and then predicted its corresponding signaling pathways (KEGG-system) with gene ontology.

\section{Specimens}

A total of 126 lung cancer tissue samples were collected from Kaohsiung Medical University Hospital (from 1991 to 2007) after obtaining IRB approval (KMUH-IRB-20110286). The histological diagnosis and grade of lung cancer were determined according to the WHO classification. The clinicopathological features, including the tumor size, local invasion, lymph node involvement, and distal metastases, were determined according to the AJCC TNM classification of lung cancer ${ }^{3}$. The follow-up duration was up to 200 months. The study employed a retrospective design, and only archived surgically removed tumor samples embedded in paraffin were used; thus, we did not obtain a registration number from the ClinicalTrials.gov website. The requirement for informed consent was waived by the Institutional Review Board of Kaohsiung Medical University Hospital of Taiwan.

\section{Immunohistochemistry}

Immunohistochemical (IHC) staining was performed using the Ventana IHC staining system (Ventana, Tucson, AZ). IHC was performed using the primary antibodies against SQS (1:100, GTX104091, Genetex) and OPN (1:1000, ab69498, Abcam). The OPN score was based on the intensity of IHC staining: - , negative; $1+, 0-20 \%$ of tumor cell stained; $2+, 20-50 \%$ of tumor cell stained; and $3+,>50 \%$ of tumor cell stained.

\section{Statistical methods}

Student's $t$ test was used to analyze the statistical significance of the results obtained from least three independent experiments, and the data are presented as the means \pm SDs. Differences in the clinicopathological characteristics were determined using the chi-square test. Survival curves were analyzed using the log-rank test (generated using the Kaplan-Meier method). Univariate and multivariate models were established using a Cox proportional hazards regression analysis to evaluate the prognostic significance of various factors. All statistical tests were two-sided. $P<0.05$ was considered significant. Statistical analyses were performed using SPSS (Statistical Package for the Social Sciences, version 19.0) software.

\section{Acknowledgements \\ This study was supported by grants from the National Science Council [MOST 107-2320-B-001 -016 -MY3], [MOST-108-3114-Y-001-002], Academia Sinica [AS- SUMMIT-108], [AS-SUMMIT-109], [AS-KPQ-109-BioMed] to M.H. and Kaohsiung Veterans General Hospital, Taiwan [VGHKS109-176] to Y.F.Y. The authors would like to thank the GRC Instrument Core Facilities for their support for the Aperio digital pathology analyses.}

\begin{abstract}
Author details
${ }^{1}$ Department of Medical Education and Research, Kaohsiung Veterans General Hospital, Kaohsiung, Taiwan. ${ }^{2}$ Department of Biomedical Imaging and Radiological Sciences, National Yang-Ming University, Taipei, Taiwan. ${ }^{3}$ Genomics Research Center, Academia Sinica, Taipei, Taiwan. ${ }^{4}$ Division of Pulmonary and Critical Care Medicine, Department of Internal Medicine, Kaohsiung Medical University Hospital, Kaohsiung Medical University, Kaohsiung, Taiwan. ${ }^{5}$ Department of Internal Medicine, Kaohsiung Municipal TaTung Hospital, Kaohsiung Medical University, Kaohsiung, Taiwan. 'Department of Respiratory Therapy, College of Medicine, Kaohsiung Medical University, Kaohsiung, Taiwan. ${ }^{7}$ Department of Internal Medicine E-DA Cancer Hospital School of Medicine, I-Shou University Kaohsiung, Kaohsiung, Taiwan. ${ }^{8}$ Department of Biochemistry, College of Medicine, Kaohsiung Medical University, Kaohsiung, Taiwan
\end{abstract}

\section{Author contributions}

The authors contributed in the following way: design and write the manuscripts: Y.-F.Y., Y.-C.C., and M.H.; provide materials: C.-J.Y., M.-S.H., and M.H.; perform experiments: Y.-F.Y. and Y.-H.J.; interpret data and supervision: Y.-C.C. and M.H.

Conflict of interest

The authors declare that they have no conflict of interest. 


\section{Publisher's note}

Springer Nature remains neutral with regard to jurisdictional claims in published maps and institutional affiliations.

Supplementary Information accompanies this paper at (https://doi.org/ 10.1038/s41389-020-00262-2).

Received: 27 February 2020 Revised: 3 August 2020 Accepted: 11 August 2020

Published online: 29 August 2020

\section{References}

1. Parkin, D. M., Bray, F., Ferlay, J. \& Pisani, P. Global cancer statistics, 2002. CA Cancer J. Clin. 55, 74-108 (2005).

2. Ihde, D. C. Chemotherapy of lung cancer. N. Engl. J. Med 327, 1434-1441 (1992).

3. Hoffman, P. C., Mauer, A. M. \& Vokes, E. E. Lung cancer. Lancet $\mathbf{3 5 5}, \mathbf{4 7 9 - 4 8 5}$ (2000).

4. Parkin, D. M. Global cancer statistics in the year 2000. Lancet Oncol. 2, 533-543 (2001).

5. Spira, A. \& Ettinger, D. S. Multidisciplinary management of lung cancer. N. Engl. J. Med. 350, 379-392 (2004).

6. Herbst, R. S., Heymach, J. V. \& Lippman, S. M. Lung cancer. N. Engl. J. Med. 359 1367-1380 (2008).

7. Yang, Y. F. et al. Squalene synthase induces tumor necrosis factor receptor 1 enrichment in lipid rafts to promote lung cancer metastasis. Am. J. Respir. Crit. Care Med. 190, 675-687 (2014).

8. Silvius, J. R., del Giudice, D. \& Lafleur, M. Cholesterol at different bilayer concentrations can promote or antagonize lateral segregation of phospholipids of differing acyl chain length. Biochemistry 35, 15198-15208 (1996).

9. Garman, S. C., Kinet, J. P. \& Jardetzky, T. S. Crystal structure of the human highaffinity lgE receptor. Cell 95, 951-961 (1998).

10. Suzuki, K. G. et al. GPI-anchored receptor clusters transiently recruit Lyn and G alpha for temporary cluster immobilization and Lyn activation: singlemolecule tracking study 1. J. Cell Biol. 177, 717-730 (2007).

11. Tran, D., Carpentier, J. L., Sawano, F., Gorden, P. \& Orci, L. Ligands internalized through coated or noncoated invaginations follow a common intracellular pathway. Proc. Natl Acad. Sci. USA 84, 7957-7961 (1987).

12. Sheets, E. D., Holowka, D. \& Baird, B. Critical role for cholesterol in Lynmediated tyrosine phosphorylation of FcepsilonRl and their association with detergent-resistant membranes. J. Cell Biol. 145, 877-887 (1999).

13. Rangaswami, H., Bulbule, A. \& Kundu, G. C. Osteopontin: role in cell signaling and cancer progression. Trends Cell Biol. 16, 79-87 (2006).

14. Senger, D. R., Wirth, D. F. \& Hynes, R. O. Transformed mammalian cells secrete specific proteins and phosphoproteins. Cell 16, 885-893 (1979).

15. El-Tanani, M. K. Role of osteopontin in cellular signaling and metastatic phenotype. Front. Biosci. J. virtual Libr. 13, 4276-4284 (2008).

16. Chu, Y. W. et al. Selection of invasive and metastatic subpopulations from a human lung adenocarcinoma cell line. Am. J. Respir. Cell Mol. Biol. 17, 353-360 (1997).

17. Meyers, R. M. et al. Computational correction of copy number effect improves specificity of CRISPR-Cas9 essentiality screens in cancer cells. Nat. Genet. 49, 1779-1784 (2017).

18. Li, H. et al. The landscape of cancer cell line metabolism. Nat. Med. 25, 850-860 (2019).

19. Gniadecki, R. Depletion of membrane cholesterol causes ligand-independent activation of Fas and apoptosis. Biochem Biophys. Res Commun. 320, 165-169 (2004).

20. Carpenter, G. The EGF receptor: a nexus for trafficking and signaling. Bioessays 22, 697-707 (2000)

21. Muppidi, J. R. \& Siegel, R. M. Ligand-independent redistribution of Fas (CD95) into lipid rafts mediates clonotypic T cell death. Nat. Immunol. 5, 182-189 (2004).

22. Harder, T. Lipid raft domains and protein networks in T-cell receptor signal transduction. Curr. Opin. Immunol. 16, 353-359 (2004).

23. Waugh, M. G., Minogue, S., Anderson, J. S., dos Santos, M. \& Hsuan, J. J. Signalling and non-caveolar rafts. Biochem Soc. Trans. 29, 509-511 (2001).

24. Shenoy-Scaria, A. M., Dietzen, D. J., Kwong, J., Link, D. C. \& Lublin, D. M. Cysteine3 of Src family protein tyrosine kinase determines palmitoylation and localization in caveolae. J. Cell Biol. 126, 353-363 (1994).
25. Gyorffy, B., Surowiak, P., Budczies, J. \& Lanczky, A. Online survival analysis software to assess the prognostic value of biomarkers using transcriptomic data in non-small-cell lung cancer. PLoS One 8, e82241 (2013).

26. Shan, M. et al. Cigarette smoke induction of osteopontin (SPP1) mediates $\mathrm{T}(\mathrm{H})$ 17 inflammation in human and experimental emphysema. Sci. Transl. Med. 4, 117 ra119 (2012)

27. Reppert, S., Koch, S. \& Finotto, S. IL-17A is a central regulator of lung tumor growth. Oncoimmunology 1, 783-785 (2012).

28. Durham, A. L. \& Adcock, I. M. The relationship between COPD and lung cancer. Lung Cancer 90, 121-127 (2015).

29. $\mathrm{Hu}$, Z. et al. Overexpression of osteopontin is associated with more aggressive phenotypes in human non-small cell lung cancer. Clin. Cancer Res. Off. J. Am. Assoc. Cancer Res. 11, 4646-4652 (2005).

30. Blasberg, J. D. et al. Reduction of elevated plasma osteopontin levels with resection of non-small-cell lung cancer. J. Clin. Oncol. Off. J. Am. Soc. Clin. Oncol. 28, 936-941 (2010)

31. Kaomongkolgit, R., Manokawinchoke, J., Sanchavanakit, N., Pavasant, P. \& Sumrejkanchanakij, P. Fibronectin supports TNF-alpha-induced osteopontin expression through beta1 integrin and ERK in HN-22 cells. Arch. Oral. Biol. 55, 101-107 (2010).

32. Senger, D. R., Perruzzi, C. A., Papadopoulos-Sergiou, A. \& Van de Water, L. Adhesive properties of osteopontin: regulation by a naturally occurring thrombin-cleavage in close proximity to the GRGDS cell-binding domain. Mol. Biol. Cell 5, 565-574 (1994).

33. Das, R., Mahabeleshwar, G. H. \& Kundu, G. C. Osteopontin induces AP-1mediated secretion of urokinase-type plasminogen activator through c-Srcdependent epidermal growth factor receptor transactivation in breast cancer cells. J. Biol. Chem. 279, 11051-11064 (2004).

34. Tuck, A. B., Hota, C., Wilson, S. M. \& Chambers, A. F. Osteopontin-induced migration of human mammary epithelial cells involves activation of EGF receptor and multiple signal transduction pathways. Oncogene 22, 1198-1205 (2003).

35. Beck, G. R. Jr. \& Knecht, N. Osteopontin regulation by inorganic phosphate is ERK1/2-, protein kinase C-, and proteasome-dependent. J. Biol. Chem. 278, 41921-41929 (2003)

36. Tezuka, K., Denhardt, D. T., Rodan, G. A. \& Harada, S. Stimulation of mouse osteopontin promoter by $\mathrm{v}$-Src is mediated by a CCAAT box-binding factor. J. Biol. Chem. 271, 22713-22717 (1996).

37. $\mathrm{Yu}, \mathrm{X}$. et al. Osteopontin promotes hepatocellular carcinoma progression via the PI3K/AKT/Twist signaling pathway. Oncol. Lett. 16, 5299-5308 (2018).

38. Oneyama, C. et al. Transforming potential of Src family kinases is limited by the cholesterol-enriched membrane microdomain. Mol. Cell Biol. 29, 6462-6472 (2009).

39. Holzer, R. G. et al. Saturated fatty acids induce c-Src clustering within membrane subdomains, leading to JNK activation. Cell 147, 173-184 (2011).

40. Zeng, J. et al. Aggregation of lipid rafts activates c-met and c-Src in non-small cell lung cancer cells. BMC Cancer 18, 611 (2018).

41. Hitosugi, T., Sato, M., Sasaki, K. \& Umezawa, Y. Lipid raft specific knockdown of SRC family kinase activity inhibits cell adhesion and cell cycle progression of breast cancer cells. Cancer Res. 67, 8139-8148 (2007).

42. Kassenbrock, C. K., Hunter, S., Garl, P., Johnson, G. L. \& Anderson, S. M. Inhibition of Src family kinases blocks epidermal growth factor (EGF)-induced activation of Akt, phosphorylation of c-Cbl, and ubiquitination of the EGF receptor. J. Biol. Chem. 277, 24967-24975 (2002).

43. Hojo, Y. et al. Matrix metalloproteinase-1 expression by interaction between monocytes and vascular endothelial cells. J. Mol. Cell Cardiol. 32, 1459-1468 (2000).

44. Rodriguez-Viciana, P. et al. Phosphatidylinositol-3-OH kinase as a direct target of Ras. Nature 370, 527-532 (1994).

45. Pacold, M. E. et al. Crystal structure and functional analysis of Ras binding to its effector phosphoinositide 3-kinase gamma. Cell 103, 931-943 (2000).

46. Jeon, J. H. et al. Lipid raft modulation inhibits NSCLC cell migration through delocalization of the focal adhesion complex. Lung Cancer 69, 165-171 (2010).

47. Murai, T. et al. Low cholesterol triggers membrane microdomain-dependent CD44 shedding and suppresses tumor cell migration. J. Biol. Chem. 286, 1999-2007 (2011).

48. Lin, Y. H. \& Yang-Yen, H. F. The osteopontin-CD44 survival signal involves activation of the phosphatidylinositol 3-kinase/Akt signaling pathway. J. Biol. Chem. 276, 46024-46030 (2001).

49. Chellaiah, M. A. et al. Osteopontin deficiency produces osteoclast dysfunction due to reduced CD44 surface expression. Mol. Biol. Cell 14, 173-189 (2003).

50. Rud, A. K. et al. Osteopontin is a prognostic biomarker in non-small cell lung cancer. BMC Cancer 13, 540 (2013). 
51. Lee, J. L. et al. Osteopontin promotes integrin activation through outside-in and inside-out mechanisms: OPN-CD44V interaction enhances survival in gastrointestinal cancer cells. Cancer Res. 67, 2089-2097 (2007).

52. Lee, J. L., Wang, M. J., Sudhir, P. R. \& Chen, J. Y. CD44 engagement promotes matrix-derived survival through the CD44-SRC-integrin axis in lipid rafts. Mol. Cell Biol. 28, 5710-5723 (2008).

53. Wai, P. Y. \& Kuo, P. C. The role of Osteopontin in tumor metastasis. J. Surg. Res. 121, 228-241 (2004).

54. Tuck, A. B., Elliott, B. E., Hota, C., Tremblay, E. \& Chambers, A. F. Osteopontininduced, integrin-dependent migration of human mammary epithelial cells involves activation of the hepatocyte growth factor receptor (Met). J. Cell Biochem 78, 465-475 (2000).

55. Li, N. Y. et al. Osteopontin up-regulates critical epithelial-mesenchymal transition transcription factors to induce an aggressive breast cancer phenotype. J. Am. Coll. Surg. 217, 17-26 (2013). discussion 26.

56. Raja, R. et al. Hypoxia-driven osteopontin contributes to breast tumor growth through modulation of HIF1alpha-mediated VEGF-dependent angiogenesis. Oncogene 33, 2053-2064 (2014).
57. Angelucci, A. et al. Osteopontin enhances the cell proliferation induced by the epidermal growth factor in human prostate cancer cells. Prostate 59, 157-166 (2004).

58. Shi, L. \& Wang, X. Role of osteopontin in lung cancer evolution and heterogeneity. Semin Cell Dev. Biol. 64, 40-47 (2017).

59. Bendeck, M. P. et al. Smooth muscle cell matrix metalloproteinase production is stimulated via alpha(v)beta(3) integrin. Arterioscler Thromb. Vasc. Biol. 20, 1467-1472 (2000).

60. Cheng, H. C. et al. Loss of RUNX3 increases osteopontin expression and promotes cell migration in gastric cancer. Carcinogenesis 34, 2452-2459 (2013).

61. Park, S., Jung, H. H., Park, Y. H., Ahn, J. S. \& Im, Y. H. ERK/MAPK pathways play critical roles in EGFR ligands-induced MMP1 expression. Biochem Biophys. Res Commun. 407, 680-686 (2011).

62. Huang, D. W. et al. The DAVID Gene Functional Classification Tool: a novel biological module-centric algorithm to functionally analyze large gene lists. Genome Biol. 8, R183 (2007). 